\title{
Graphene Spintronic Devices with Molecular Nanomagnets
}

\author{
Andrea Candini, ${ }^{*,+, \|}$ Svetlana Klyatskaya, ${ }^{\ddagger}$ Mario Ruben, ${ }^{,, \S}$ Wolfgang Wernsdorfer," and Marco Affronte ${ }^{+, \perp}$ \\ ${ }^{+}$Centro S3, Istituto Nanoscienze-CNR, via Campi 213/a, 41125 Modena, Italy \\ ${ }^{\ddagger}$ Institute of Nanotechnology (INT), Karlsruhe Institute of Technology (KIT), 76344 Eggenstein-Leopoldshafen, Germany \\ ${ }^{\S}$ IPCMS CNRS_Université de Strasbourg, 67034 Strasbourg, France \\ "Institut Néel, CNRS et Université Joseph Fourier, BP 166, F-38042 Grenoble Cedex 9, France \\ ${ }^{\perp}$ Dipartimento di Fisica, Università di Modena e Reggio Emilia, via Campi 213/a, 41125 Modena, Italy \\ Supporting Information
}

ABSTRACT: The possibility to graft nano-objects directly on its surface makes graphene particularly appealing for device and sensing applications. Here we report the design and the realization of a novel device made by a graphene nanoconstriction decorated with $\mathrm{TbPc}_{2}$ magnetic molecules ( $\mathrm{Pc}=$ phthalocyananine), to electrically detect the magnetization reversal of the molecules in proximity with graphene. A magnetoconductivity signal as high as $20 \%$ is found for the spin reversal, revealing the uniaxial magnetic anisotropy of the $\mathrm{TbPc}_{2}$ quantum magnets. These results depict the behavior of multiple-field-effect nanotransistors with sensitivity at the single-molecule level.

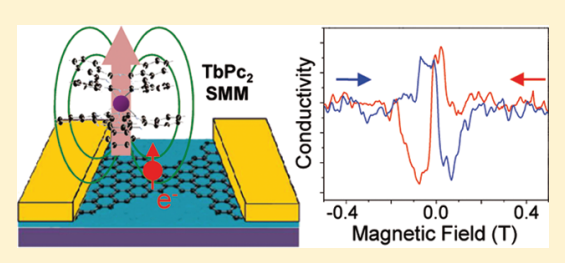

KEYWORDS: Single-molecule magnets, graphene nanodevices, single-molecule detection, molecular spintronics

Cingle-molecule magnets (SMMs) have been demonstrated to $\checkmark$ possess well-defined quantum states and quantum behavior at the single molecule level. ${ }^{1}$ The presence of a high-spin state exhibiting a magnetic anisotropy barrier leads to the observation of quantum tunneling of the magnetization (QTM). Very recently, observation of QTM by X-ray magnetic dichroism was reported for monolayers of isolated $\mathrm{Fe}_{4}$ SMMs on a $\mathrm{Au}(111)$ substrate. ${ }^{2}$ The crossing between two molecular levels can also be studied at finite field and tuned in different crystallographic directions in the laboratory. ${ }^{3}$ While these issues have been intensively studied in ensembles of identical molecules, the observation of the magnetization reversal on one or very-few molecules has not been reported yet.

Going beyond magnetic effects, the control of magnetic molecules as active units in a spintronic device was recently proposed. ${ }^{4,5}$ In particular, the class of molecular quantum magnets offers the spin degree that can be used to control the charge transport in conducting systems. $s p^{2}$ carbon substrates, such as graphene ${ }^{6}$ or carbon nanotubes, ${ }^{7}$ have been proven to act as an integrating base for embedding nanoscale molecular properties into device environments, due to their particular combination of high $2 \mathrm{D}$ and $1 \mathrm{D}$ aspect ratios with unique conductivity properties, such as bipolar tunability of charge and high mobility. With such approaches, tiny detectors, including magnetic flux carbon nanotube (CNT) nanoSQUIDs, have been fabricated. ${ }^{8}$ An important feature of carbon-based nanostructured materials is also the direct exposure of the active-conducting - area to the external world. Finally, in view of spintronic devices, $s p^{2}$ carbon substrates are of particular interest due to the expected long spin coherence lifetimes and lengths. ${ }^{9}$

Herein we present the design, realization, and characterization of a novel hybrid spintronic nanodevice (see Figure 1) made by the integration of a graphene nanoconstriction, working in the
Coulomb blockade regime, and terbium(III) bis(phthalocyanine) SMMs (see Figure 1a,b). Low-temperature experiments show evidence for the electrical detection of the magnetization reversal of the $\mathrm{TbPc}_{2} \mathrm{SMMs}_{\mathrm{s}}$ through the parallel (external magnetic field applied in the graphene plane) magnetoconductivity.

Our pristine devices consist of a few nanometers wide graphene nanoconstriction obtained by two steps of electron beam lithography (EBL) and oxygen plasma etching. Graphene flakes are obtained by the standard mechanical exfoliation method on top of a p-doped silicon wafer (used as the backgate) coated with $300 \mathrm{~nm}$ of oxide. The effective number of layers is determined by optical contrast and checked by micro-Raman spectroscopy (see also the Supporting Information). EBL is used to deposit metal contacts $(\mathrm{Ti} / \mathrm{Pt} 10 / 100 \mathrm{~nm})$ on the graphene sheets and to pattern the device with the desired geometry. To remove unwanted regions of graphene, we used $1 \mathrm{~s}$ of exposure of oxygen plasma at $50 \mathrm{~W}$. The resulting devices are similar to the one presented in Figure 1d, where a scanning electron microscopy (SEM) image is presented. Electrical measurements are performed by using the lock-in technique with an applied ac voltage $(<100 \mu \mathrm{V} @ 33 \mathrm{~Hz})$ in a dilution fridge operated at a base temperature of $40 \mathrm{mK}$ and equipped with a $3 \mathrm{D}$ vector magnet with sweeping rates as fast as $0.2 \mathrm{~T} / \mathrm{s}$.

The low-temperature zero-bias differential conductivity $G$ is shown in Figure 2a. The device has an insulating behavior for all the accessible gate regions, except for few resonances, in agreement with what was reported for similar systems. ${ }^{10-13}$ The differential conductivity $G$ plotted as a function of both backgate voltage $V_{\mathrm{bg}}$ and the source-drain bias $V_{\mathrm{SD}}$ (Figure 2b), exhibits

Received: February 22, 2011

Revised: $\quad$ May 4, 2011

Published: June 07, 2011 


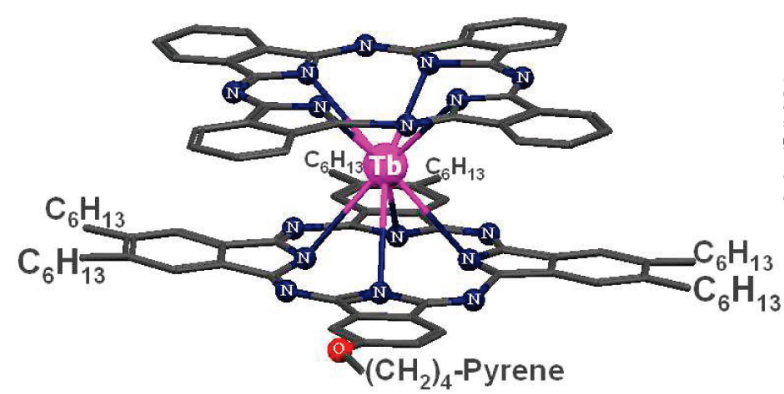

C

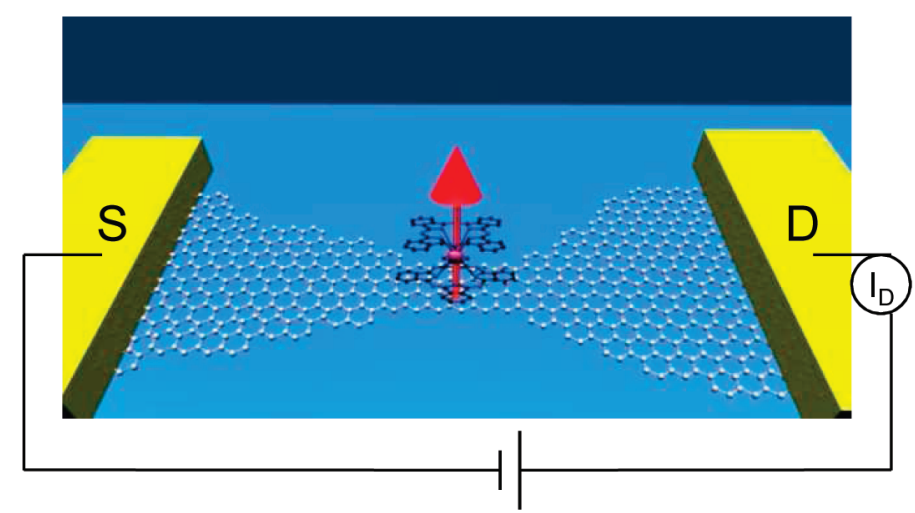

b

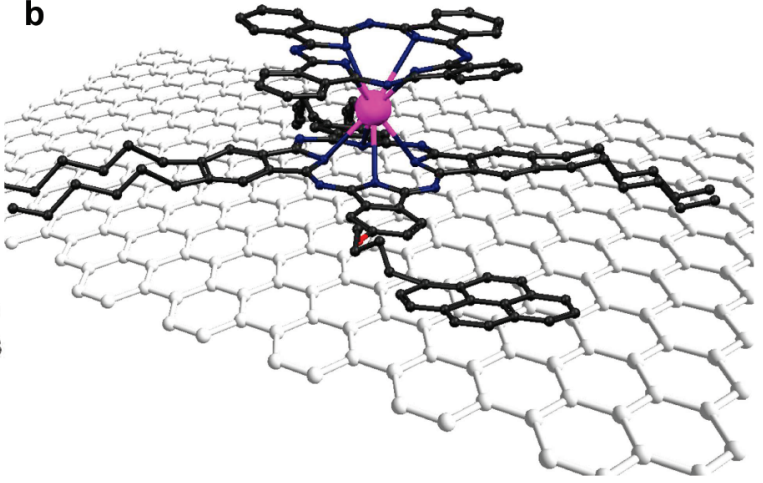

d

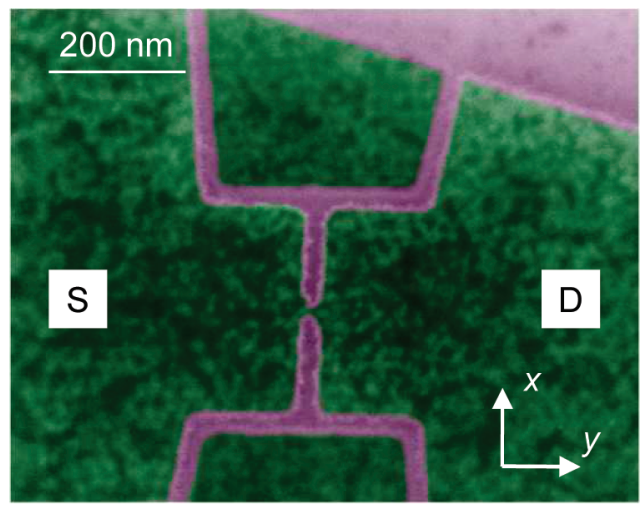

Figure 1. (a) Schematic representation of the $\mathrm{TbPc}_{2}$ single molecule magnet (SMM). (b, c) Schematic view of the device, showing in (b) the molecule attached to graphene and in (c) the nanoconstriction contacted by source $(S)$ and drain $(D)$ electrodes. The magnetic moments of the TbPc $\mathrm{S}_{2} \mathrm{SMMs}_{\mathrm{s}}$ (hexyl and pyrenyl groups here omitted for clarity) on top of the constriction add another degree of freedom to tune the conductivity of the device. (d) False-color SEM image of the device presented in the text. $\mathrm{SiO}_{2}$ substrate and etched graphene are colored in purple. Graphene conductive regions are colored in green. Source and drain electrodes are indicated. Coordinate axis are indicated as referred in the text.

typical Coulomb diamond-like characteristics. The charge transport in such a device can be modeled by one or a few quantum dots in series ${ }^{11-13}$ in which electrons tunnel through discrete levels, due to both the reduced size and the charging energy $E_{C}$ of the dot capacitance. As largely reported in literature for similar systems, ${ }^{11-17}$ the formation of a quantum dot is ascribed to the presence of edge roughness, which creates a disordered potential along the nanoconstriction in the presence of a confinement gap. Under these conditions, the dot energy levels are spatially localized in conductive islands inside the constriction, separated by tunnel barriers made by disordered graphene. The height of the Coulomb diamond, measured along the voltage bias axis, provides a charging energy of $E_{\mathrm{C}} \sim 35 \mathrm{meV}$ and thus the actual size of the dot. According to the work by Ponomarenko et al., ${ }^{10}$ the dot size scales as $d \approx 500 \mathrm{mV} \mathrm{nm} / E_{\mathrm{c}}$, yielding $\sim 15-20 \mathrm{~nm}$ for our case, in agreement with the SEM picture shown in Figure 1b. Recently, there was an important advance in the fabrication and comprehension of graphene quantum dots: in particular, the sensitivity of the conductivity $G$ to variation in the electrostatic environment ${ }^{18}$ and to the orbital and spin states of conducting electrons has been reported. ${ }^{19}$

If a magnetic field is applied within the graphene sheet, finite magnetoconductivity appears only for backgate voltage values that drive the nanoconstriction to conductivity resonances. This effect offers the opportunity to electrically tune the magnetoconductivity or to decouple the magnetic system from the sensor device, which would help to reduce decoherence in the system.
The pyrene-substituted terbium(III) bis(phthalocyanine) single-molecule magnets $\left(\mathrm{TbPc}_{2} \text { hereafter, see Figure } 1 \mathrm{a}\right)^{20,21}$ are synthesized as reported before ${ }^{20}$ and are deposited from the liquid phase. Concentrations in dichloromethane $(\mathrm{dcm})$ less than $10^{-6} \mathrm{~mol} \cdot \mathrm{L}^{-1}$ have been employed to avoid agglomeration of molecules. Immediately before molecule deposition, the devices are thermal annealed under Ar flux at $300{ }^{\circ} \mathrm{C}$ to remove residues from the lithography processes. On the basis of our previous work ${ }^{21}$ and on the molecular geometry, ${ }^{20}$ an averaged SMM density of $\sim 10$ molecules can be estimated for the herein presented graphene nanoconstriction. Combined Raman and conductance studies at room temperature show that the electronic properties of the graphene sheets remain intact after SMMs deposition for such low concentrations. ${ }^{21}$ Also at low temperatures and in zero applied magnetic field, the electrical features of the nanoconstrictions are not altered by the deposition of molecules. Recent studies have shown that unsubstituted $\mathrm{TbPc}_{2}$ molecules adsorb flat lying on a $\mathrm{Cu}(111)$ surface, with the phthalocyanine planes parallel to the surface plane. ${ }^{22}$ Similar results have been recently reported for $\mathrm{TbPc}_{2}$ on $\mathrm{Au}(111),{ }^{23}$ on $\mathrm{Cu}(100),{ }^{24}$ and on HOPG. ${ }^{25}$ However, due to the corrugation of the graphene surface and the presence of ripples, the orientation of the pyrene substituted $\mathrm{TbPc}_{2}$ SMMs can statistically present some misalignment leading to a finite tilt angle between the main device plane and the Pc plane. Concerning the magnetic properties of $\mathrm{TbPc}_{2}$, due to the presence of the ligand field, the $\mathrm{Tb}$ (III) ion possess a split $J=6$ ground multiplet with $J_{z}= \pm 6$ sublevels 


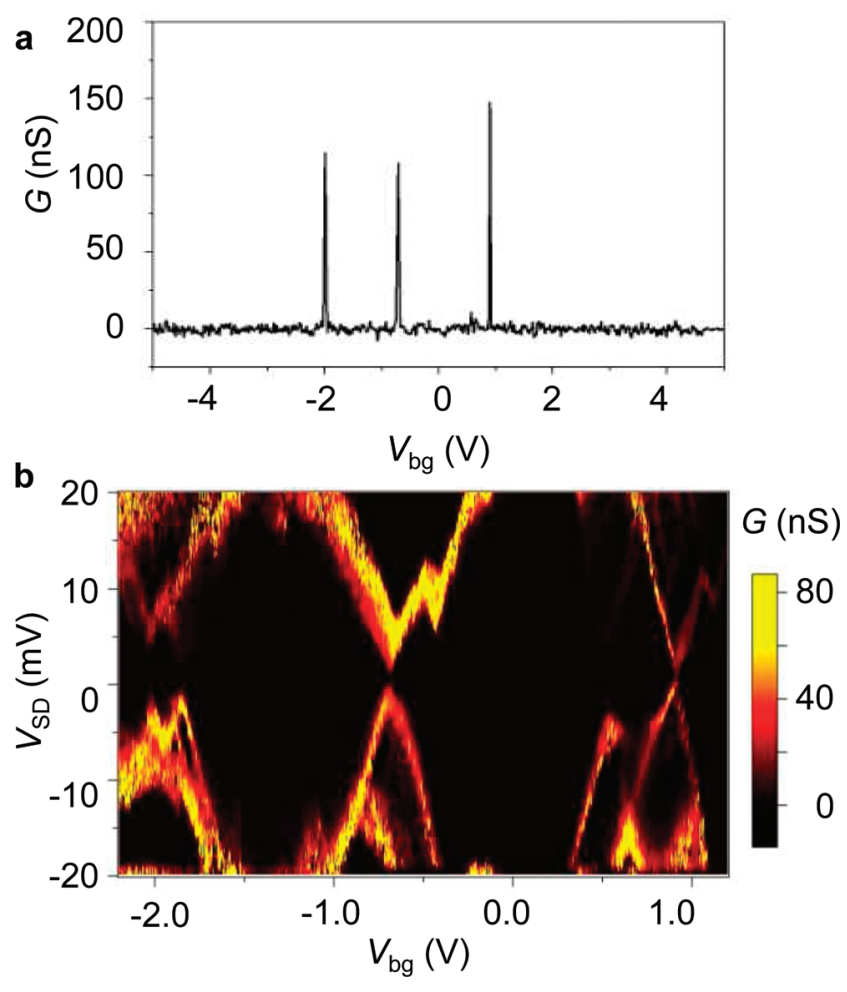

Figure 2. (a) Differential conductivity $G$ vs backgate voltage $V_{\mathrm{bg}}$. The conductivity is always below the limit of detection $\left(G<10^{-9} \mathrm{~S}\right)$, apart from few resonance. The same behavior is found along all the scanned gate region, between -20 and $+20 \mathrm{~V}$. (b) Color scale plot of the differential conductivity $G$ vs backgate voltage $V_{\mathrm{bg}}$ and source-drain bias $V_{\text {sd. }}$ The appearance of the characteristic Coulomb diamonds indicates that the transport is in the Coulomb blockade regime. The typical charging energy for the largest diamonds is $E_{\mathrm{c}} \sim 35 \mathrm{meV}$.

well isolated from excited states $\left(J_{z}= \pm 5\right.$ are more than $600 \mathrm{~K}$ above the ground state doublet) and an uniaxial magnetic anisotropy, ${ }^{26}$ which is oriented perpendicularly to the phthalocyanine planes, ${ }^{22}$ resulting in an overall high energy barrier. An avoided level crossing between the $J_{z}= \pm 6$ sublevels is found at zero field, while multiple level anticrossing occurs between -0.05 and $0.05 \mathrm{~T}$, due to the hyperfine interaction with the $I=3 / 2 \mathrm{~Tb}$ nuclear spin, opening several possible tunneling resonances. ${ }^{27}$ Further transitions between the $J_{z}= \pm 6$ sublevels may occur at higher fields mediated by phonons (the so-called direct relaxation process). The magnetization behavior of $\mathrm{TbPc}_{2}$ observed in bulk crystals presents therefore the following fingerprints: (i) a strong Ising-type anisotropy; (ii) molecules that reverse their spin around zero field via tunneling; (iii) phonon-mediated direct transitions at higher fields. In addition, the hysteresis loop depends on the field sweep rate, as the probability that a molecule reverses its magnetization at a certain level crossing is given by the Landau-Zener mechanism: ${ }^{28}$ it is observed that the magnetization reversal starts at more negative fields for the slower rates, and the hysteresis loop is broadened around zero field for the fast rates. These characteristics were recently observed also on $\mathrm{TbPc}_{2}$ monolayers by XMCD measurements, ${ }^{22,29,30}$ although hysteresis may be quenched at increased temperatures by the interaction with the surface ${ }^{29}$ and depends on duration/method of measurement. ${ }^{22,30}$

The effects of the molecule grafting on our graphene devices become visible when a magnetic field is applied, as shown in

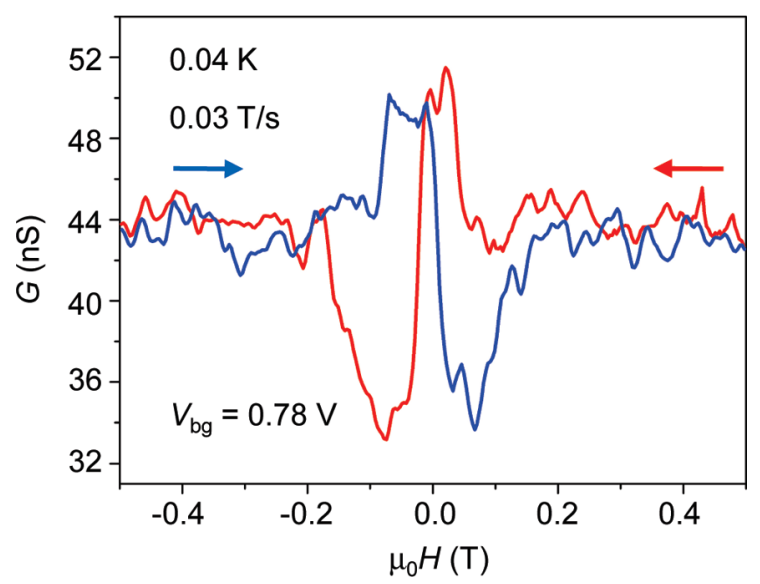

Figure 3. Zero-bias differential magnetoconductivity of the device graphene plus $\mathrm{TbPc}_{2} \mathrm{SMM}$ obtained at a fixed backgate voltage $\left(V_{\mathrm{bg}}\right.$ is fixed at a conducting resonance). The blue curve represents the magnetoconductivity under increasing field (trace), while for the red curve the field is decreasing (retrace). The observed hysteresis is given by the magnetization reversal of the TbPc2 SMMs deposited on the graphene constrictions.

Figure 3. We have characterized more than 20 hybrid devices in the Coulomb blockade regime and only in a few of them significant deviations from the empty devices were detected, indicating that the efficiency of molecular grafting is rather low at this concentration. In the following we focus on one device on which we obtained a full series of results (see also the Supporting Information). The magnetoconductivity curve $G(H)$, obtained at a fixed backgate voltage $V_{\mathrm{bg}}$ for the field applied in the plane of graphene, shows the opening of a hysteresis loop (Figure 3). Starting from negative field, the conductivity has initially the value $G_{\text {sat }}$, then it displays a positive bump for small negative magnetic field values, and it abruptly drops crossing zero field to be finally restored at its initial value $G_{\text {sat }}$ for increasing positive fields. A symmetric behavior is observed with the magnetic field swept in the opposite direction. The curve shown in Figure 3 is obtained on a single run (one sweep up followed by one sweep down) but the main features are well reproducible. The abrupt change in $G(H)$ crossing zero magnetic field as well as the restoring of initial value $G_{\text {sat }}$ at sufficiently high fields ( $>0.2 \mathrm{~T}$ in Figure 3$)$ are repeatedly observed upon multiple field sweeping.

The difference between the up-sweep (trace) and the downsweep (retrace) $G(H)$ curves, indicated as $\delta G(H)$ hereafter, can be used to characterize the hysteresis cycle. We notice that $\delta G(H)$ values can be as high as $\sim 20 \%$ of the initial conductivity. The hysteresis loop is reproducibly found (with the same sign) along the conductivity resonances shown in Figure 2a, while it vanishes as soon as $V_{\mathrm{bg}}$ is tuned out of the resonance peaks (see also the Supporting Information).

In Figure 4 the magnetoconductivity curves for different magnetic field sweep rates are depicted. The hysteresis loop and the jump of $G(H)$ close to zero field are always present but some features of the $G(H)$ curve depend on the sweeping rate: for the fastest ones, the upturn in $G(H)$ before crossing zero field appears closer to zero field and the successive drop gets broader.

Finally, Figure 5 shows the hysteresis loop dependence on the magnetic field orientation. Here $\delta G(H)$ is plotted in color code for all the field directions in the $x y$ plane of graphene. The border 


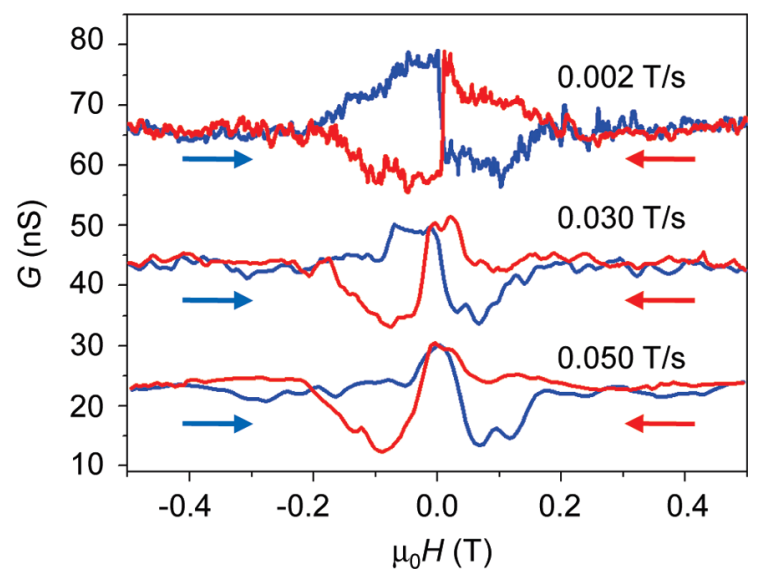

Figure 4. Differential magnetoconductivity $G$ obtained at fixed backgate voltage $V_{\mathrm{bg}}$ for different field sweep rates. For clarity, the curves taken at 0.002 and $0.05 \mathrm{~T} / \mathrm{s}$ are vertically shifted by +20 and $-20 \mathrm{nS}$, respectively. The hysteresis loops are weakly depending on the field sweep rates and remain open for very slow rates. When the field is swept very slowly, the jump of the conductivity is straighter and the hysteresis can begin even before zero field. The behavior is agreement with what is expected from magnetization measurements on crystal samples.

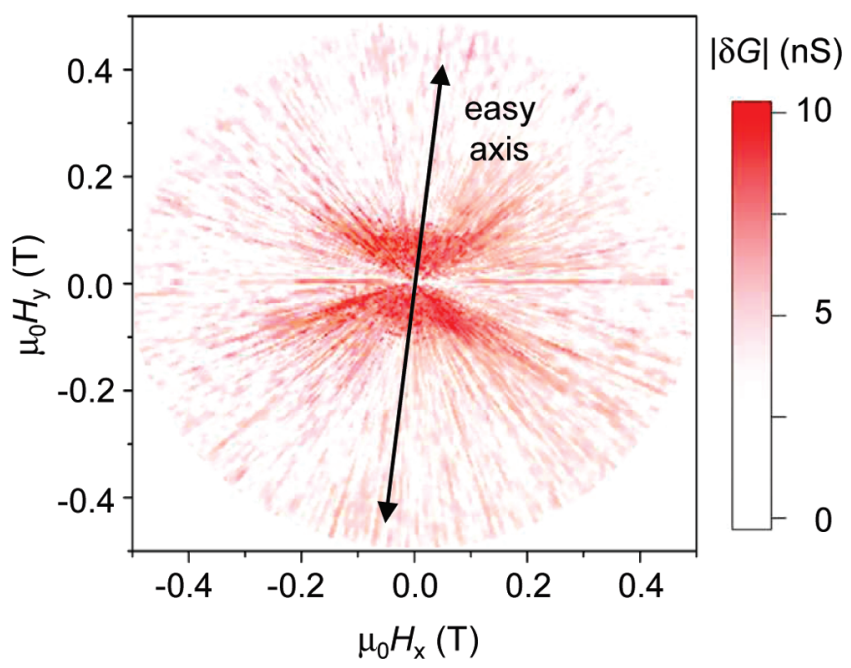

Figure 5. Color plot of $\delta G(H)$ (difference between trace and retrace) for all directions of the magnetic field in the plane of graphene. The border between the red regions and the noncolored ones represents the fields at which the hysteresis loop is closing. The behavior is typical for magnetic systems with uniaxial anisotropy and the easy axis is indicated. Along the hard direction (perpendicular to the easy axis) no hysteresis is observed as the magnetic field is not high enough to reverse the magnetization of the system.

between the red and the noncolored regions represents the field $H_{\mathrm{c}}$ at which the hysteresis loop closes (i.e., $G(H)$ recovers the initial $G_{\text {sat }}$ value). We notice that $H_{\mathrm{c}}$ strongly depends on the orientation: it is minimum at an angle of $\sim 15^{\circ}$ with respect to the $y$ direction (see Figure 1d for the definition of the $x$ and $y$ axis), while it constantly increases (considering the absolute value) away from this direction. Around an angle of $-15^{\circ}$ with respect to the $x$ direction, no hysteresis is observed. This behavior is typical for magnetic systems with uniaxial anisotropy.

The emergence of a hysteresis in the magnetoconductivity indicates the presence of "external" magnetic moments coupled with the graphene device, and it is a signature that the transport can be effectively tuned by the deposition of the $\mathrm{TbPc}_{2}$ SMMs. To unambiguously fix this point, we first distinguish the present case with the recently reported (by our group) observation of hysteresis loops in the magnetoconductivity of pristine graphene devices, ${ }^{31}$ associated to the magnetization reversal of magnetic impurities or defects present in the graphene layer. When $\mathrm{TbPc}_{2}$ magnetic molecules are intentionally deposited on devices - this work-the observed hysteresis loops present a number of differences with respect to the case associated to magnetic impurities. First, the hysteresis remains even for very slow rates; second, the hysteresis loop is strongly anisotropic, with the presence of a clear easy axis; finally, $\delta G(H)$ is not changing of sign with the backgate voltage and we always observe a drop of conductivity after crossing zero field. It is also important to note that the intensity of $\delta G(H)$ is much higher in the present case, being up to $20 \%$ of the initial conductivity, while for the case of bare graphene devices $\delta G(H)$ is around $5 \%$ for the highest sweep rates. ${ }^{31}$ We can therefore exclude that magnetic impurities embedded in graphene are the origin of the hysteresis loop shown in the present work.

Conversely, all the main characteristics of the $\mathrm{TbPc}_{2}$ hysteresis loops are found in the $G(H)$ signal of our devices. The abrupt change of $G(H)$ around $H=0$ can be associated to the fact that the $\mathrm{TbPc}_{2}$ molecules have high probability to reverse their magnetization close to zero field (see below for a more detailed description). Second, at slow sweep rates the $\mathrm{TbPc}_{2}$ molecules can reverse their magnetization even before zero field, and at higher rates the transition around $H=0$ becomes broader (Figure 4), that is what is expected because of the Landau-Zener effect. ${ }^{27,28}$ The fingerprint of the uniaxial anisotropy of the $\mathrm{TbPc}_{2}$ molecule is also observed, as shown in Figure 5. Here, the angle $15^{\circ}$ with respect to the $y$ direction indicates the direction of the "easy axis" of the molecule, while $-15^{\circ}$ with respect to the $x$ direction is the "hard" one, where no hysteresis is found since the field is not high enough to reverse the magnetization of the system.

In the following, we get more insight into the functioning of our device. In first instance, the $\mathrm{TbPc}_{2} \mathrm{SMMs}$ interact with the graphene layer through a dipolar magnetic field. $\mathrm{The} \mathrm{Tb}(\mathrm{III})$ ion, located at about $4-5 \AA$ above the graphene sheet, ${ }^{21}$ produces a stray field that can be evaluated, in a dipole approximation $\mu_{0} H \sim$ $\mu / r^{3}$ (where $\mu=g_{J} \mu_{\mathrm{B}} J$, with $J=6$ ), to be about $0.2 \mathrm{~T}$ spread in an area of about $1 \mathrm{~nm}^{2}$. Considering a random distribution of molecules on the device, the resulting magnetic field profile is highly inhomogeneous and can influence the tunneling of the conducting electrons through the barriers between the charged islands forming the graphene constriction. ${ }^{32}$ We may also consider a charge redistribution in the molecules associated to the magnetization reversal. In the Coulomb blockade regime, this will induce a change in the conductivity of the graphene channel because of capacitive coupling. The corresponding change in the conductivity should depend on the applied gate voltage and change of sign on the two sides of the Coulomb blockade peaks. This effect seems less likely in our measurements, as we always observe the same sign for the hysteresis (see also Supporting Information). Even stronger coupling between the $\mathrm{TbPc}_{2}$ SMMs and graphene may play a role. DFT calculations show that the interaction between pyrene and graphene $\left(\right.$ on $\left.\mathrm{SiO}_{2}\right)$ is noncovalent and due to weak $\pi-\pi$ and van der Waals interactions. ${ }^{21}$ However, the corrugation of graphene or the presence of defects may change this scenario and induce a 
finite orbital overlap between $\mathrm{TbPc}_{2}$ and ripples or dangling bonds in graphene. Another element worth consideration is the presence of the organic $S=1 / 2 \pi$-radical on the phthalocyaninato ring, which can get in close contact with the graphene substrate. This radical ligand state has almost the same energy as the filled, magnetically active $\mathrm{Tb} 4 \mathrm{f}$ states. $^{24}$ Furthermore, similar transition metal-Pc systems adsorbed on a graphite surface show a pinning of the lowest unoccupied molecular orbital (LUMO) level close to the Fermi level. ${ }^{25}$ These elements may therefore establish an exchange coupling between the $\mathrm{Tb}$ (III) magnetic moment and the conducting electrons of graphene.

Considering the conductivity of our device as tunneling through discrete levels localized in the constriction, the abovementioned interactions make that the tunneling probability of the electrons depends on the direction of the magnetic moment $J$ of the molecules in proximity of graphene. Indeed the $J$ state of $\mathrm{Tb}$ (III) may change either the energy level of available excited state within the dot or the spin state of such levels. Keeping these mechanisms in mind, we can now describe how the conductivity $G$ of the nanoconstriction (taking for instance the curve in Figure 3 as a reference) becomes sensitive to the magnetization reversal of the molecules. Initially, at high negative (positive) fields, conductive channels are selected by the fully polarized magnetic moments of the $\mathrm{TbPc}_{2}$ molecules. As the external field approaches zero, one or a few molecules reverse their spin, and more channels become available resulting in a bump of the conductivity. When $H$ crosses zero, the majority of the molecules flip the magnetization and a large drop in the conductivity is observed. As not yet all the molecules are polarized in the opposite direction, part of the available channels are excluded and the conductivity reaches its minimum. For further increase of the field, all the molecules finally reverse their magnetic moments, becoming again fully polarized (in the opposite direction with respect to the initial step) at $H_{c}$. The forbidden channels become accessible again, and the conductivity is restored to the initial value $G_{\text {sat }}$. Thus within this model $H_{c}$ is given by the latest molecule that reverses its magnetization, so the anisotropy plot of Figure 5 represents the switching fields of an individual molecule. As a consequence, the graphene nanoconstriction can also be employed as a sensitive magnetometer with detection capabilities down to the single-molecule level.

It is worth to point out that the scheme of hybrid nanodevices presented here possesses two controls by independent external fields: the backgate voltage $V_{\mathrm{bg}}$ and the applied magnetic field that operates independently on graphene and on the $\mathrm{TbPc}_{2}$ magnetic molecule, respectively. In principle, the realization of devices with two independent gates opens the way to the design of novel logic devices. Graphene nanocontrictions intrinsically operate as field-effect transistors since on-off conductance can be switched by the backgate voltage $V_{\mathrm{bg}}$. When $V_{\mathrm{bg}}$ is biased to a resonance conductance, the hybrid nanodevice behaves similarly to conventional spin valves ${ }^{33-35}$ as far as steps can be induced in the conductivity by reversing the external magnetic field. The scheme presented here, however, makes no use of ferromagnetic electrodes and a molecular magnetic gate is used instead, in line with what was proposed by some recent theoretical works. ${ }^{36-38}$ As future perspectives, further tunability can be obtained by employing different molecular gates with different functionalities; in addition, this scheme of device may be used to exploit the entanglement between the SMMs and graphene.

\section{ASSOCIATED CONTENT}

S Supporting Information. Details of the experimental methods, Raman characterization of the graphene flakes, more experimental data on the same device and on other devices, and study of the magnetic signal as a function of the backgate voltage. This material is available free of charge via the Internet at http:// pubs.acs.org

\section{AUTHOR INFORMATION}

\section{Corresponding Author}

*E-mail: andrea.candini@nano.cnr.it.

\section{ACKNOWLEDGMENT}

This work has been supported by FP7-ICT FET Open "MolSpinQIP" project funded by EU, Contract No. 211284, the ERC advanced grant MolNanoSpin (No. 226558), the priority program 1149 "Graphene" by the German Science Foundation (DFG), and the ANR-Pnano project MolNanoSpin. We thank E. Bonet, V. Bouchiat, T. Crozes, C. Thirion, E. Eyraud, F. Balestro, D. Lepoittevin, C. Hoarau, R. Haettel, L. Marty, N. Bendiab, M. Lopes, V. Reita, M. Urdampilleta, R. Vincent, and the Nanofab facility of Grenoble for technical support. We thank M. Rontani and F. Troiani for critical reading of the manuscript.

\section{REFERENCES}

(1) Gatteschi, D.; Sessoli, R.; Villain, J. Molecular Nanomagnets; Oxford University Press: Oxford and New York, 2006.

(2) Mannini, M.; et al. Nature 2010, 468, 417-421.

(3) Carretta, S.; et al. Phys Rev. B 2005, 72, 060403.

(4) Rocha, A. R.; et al. Nat. Mater. 2005, 4, 335-338.

(5) Bogani, L.; Wernsdorfer, W. Nat. Mater. 2008, 7, 179-186.

(6) Geim, A. K.; Novoselov, K. S. Nat. Mater. 2007, 6, 183-191.

(7) Charlier, J.-C.; Blase, X.; Roche, S. Rev. Mod. Phys. 2007, $79,677-732$.

(8) Cleuziou, J.-P.; Wernsdorfer, W.; Bouchiat, V.; Ondarçuhu, T.; Monthioux, M. Nat. Nanotechnol. 2006, 1, 53-59.

(9) Hueso, L. E.; et al. Nature 2005, 445, 410-413.

(10) Ponomarenko, E. A.; et al. Science 2008, 320, 356-358.

(11) Moser, J.; Bachtold, A. Appl. Phys. Lett. 2009, 95, 173506.

(12) Özyilmaz, B.; Jarillo-Herrero, P.; Efetov, D.; Kim., P. Appl. Phys. Lett. 2007, 91, 192107.

(13) Todd, K.; Chou, H. T.; Amasha, S.; Goldhaber-Gordon, D. Nano Lett. 2009, 9, 416-421.

(14) Stampfer, C.; et al. Phys. Rev. Lett. 2009, 102, 056403.

(15) Gallagher, P.; Todd, K.; Goldhaber-Gordon, D. Phys. Rev. B 2010, 81, 115409.

(16) Han, M. Y.; Brant, J. C.; Kim, P. Phys. Rev. Lett. 2010, $104,056801$.

(17) Sols, F.; Guinea, F.; Castro Neto, A. H. Phys. Rev. Lett. 2007, 99, 166803

(18) Wang, L. J.; et al. Appl. Phys. Lett. 2010, 97, 262113.

(19) Guttiger, J.; Frey, T.; Stampfer, C.; Ihn, T.; Ensslin, K. Phys. Rev. Lett. 2010, 105, 116801.

(20) Klyatskaya, S.; Mascarós, J. R. G.; Bogani, L.; Hennrich, F.; Kappes, M.; Wernsdorfer, W.; Ruben, M. J. Am. Chem. Soc. 2009, 131, 15143-15151.

(21) Lopes, M.; Candini, A.; Urdampilleta, M.; Reserbat-Plantey, A.; Bellini, V.; Klyatskaya, S.; Marty, L.; Ruben, M.; Affronte, M.; Wernsdorfer, W.; Bendiab, N. ACS Nano 2010, 4, 7531-7537.

(22) Stepanow, S.; Honolka, J.; Gambardella, P.; Vitali, L.; Abdurakhmanova, N.; Tseng, T.-C.; Rauschenbach, S.; Tait, S. L.; Sessi, V.; Klyatskaya, S.; Ruben, M.; Kern, K. J. Am. Chem. Soc. 2010, 132, 11900-11901. 
(23) Katoh, K.; Yoshida, Y.; Yamashita, M.; Miyasaka, H.; Breedlove, B. K.; Kajiwara, T.; Takaishi, S.; Ishikawa, N.; Isshiki, H.; Zhang, Y. F.; Komeda, T.; Yanagishi, M.; Takeya, J. J. Am. Chem. Soc. 2009, 131, 9967.

(24) Vitali, L.; Fabris, S.; Conte, A. M.; Brink, S.; Ruben, M.; Baroni, S.; Kern, K. Nano Lett. 2008, 8, 3364-3368.

(25) Gopakumar, T. G.; Müller, F.; Hietschold, M. J. Phys. Chem B 2006, 110, 6051-6065.

(26) Branzoli, F.; Carretta, P.; Filibian, M.; Zoppellaro, G.; Graf, M. J.; Galan-Mascaros, J. R.; Fuhr, O.; Brink, S.; Ruben, M. J. Am. Chem. Soc. 2009, 131, 4387-4396.

(27) Ishikawa, N.; Sugita, M.; Wernsdorfer, W. Angew. Chem., Int. Ed. 2005, 44, 2931-2935.

(28) Wernsdorfer, W.; Sessoli, R. Science 1999, 284, 133-135.

(29) Margheriti, L.; et al. Adv. Mater. 2010, 22, 5488-5493.

(30) Biagi, R; et al. Phys. Rev. B 2010, 82, 224406.

(31) Candini, A.; Alvino, C.; Wernsdorfer, W.; Affronte, M. Phys. Rev. B 2011, 83, 121401.

(32) Nogaret, A. J. Phys.: Condens. Matter 2010, 22, 253201.

(33) Hill, E., W.; Geim, A. K.; Novoselov, K. S.; Schedin, A. F.; Blake, P. IEEE Trans. Magn. 2006, 42, 2694-2696.

(34) Tombros, N.; Josza, C.; Jonkman, H. T.; van Wees, B. Nature 2007, 448, 571-574.

(35) Cho, S.; Chen, Y. S.; Fuhrer, M. S. Appl. Phys. Lett. 2007, 91, 123105 .

(36) Jayasekera, T.; Kong, B. D.; Kim, K. W.; Buongiorno Nardelli, M. Phys. Rev. Lett. 2010, 104, 146801.

(37) Semenov, Y. J.; Kim, K. W.; Zavada, J. M. Appl. Phys. Lett. 2007, 91,153105 .

(38) Haugen, H.; Huertas-Hernando, D.; Brataas, A. Phys. Rev. B 2007, 77, 115406 . 